\title{
Stress Coping Strategies among Critical Care Medicine Physicians during COVID-19 Pandemic in Egypt: A Qualitative Study
}

\author{
Rateba Said Mohammed ${ }^{1}$, Marwa Rashad Salem ${ }^{2 *}$, Ahmed Taher Mahmoud $^{3}$, Loay El Sabbahy ${ }^{4}$, \\ Shaimaa Ibrahim El-Jaafary ${ }^{5}$ \\ ${ }^{1}$ Department of Occupational and Environmental Medicine, Faculty of Medicine, Cairo University, Giza, Egypt; ${ }^{2}$ Department \\ of Public Health and Community Medicine, Faculty of Medicine, Cairo University, Giza, Egypt; ${ }^{3}$ Department of Critical Care \\ Medicine, Faculty of Medicine, Cairo University, Giza, Egypt; ${ }^{4}$ Faculty of Medicine, Cairo University Hospitals, Faculty of \\ Medicine, Cairo University, Giza, Egypt; ${ }^{5}$ Department of Neurology, Faculty of Medicine, Cairo University, Giza, Egypt
}

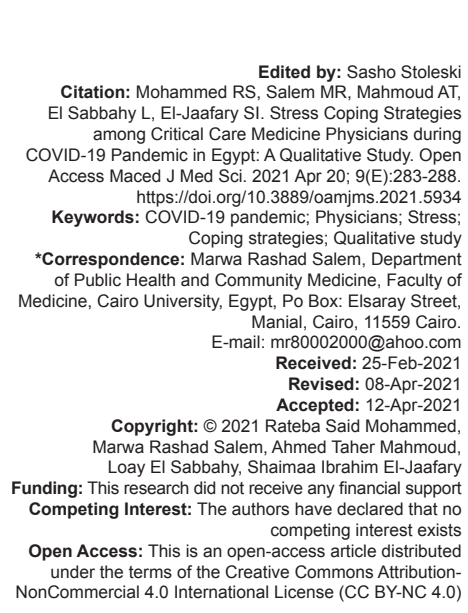

Introduction

COVID-19 is a disease that was declared by the World Health Organization (WHO) in March 2020 as a worldwide emergency [1]. The number of deaths continues to increase, with a fatality rate of $6.95 \%$, exceeding the number of severe acute respiratory syndrome (SARS) cases worldwide [2], [3]. Health care workers (HCWs) in Egypt like other medical staff worldwide are highly exposed to the COVID-19. Exposure to the new virus and its consequences together with increasing number of confirmed and suspected cases with no specific treatment, overwhelming workload, shortage of personal protective equipment (PPE), feelings of inadequate support, uncertainty and stigmatization, fear of being infected and died, or passing the infection to their families create negative emotions with high stress in the working environment that impact their physiological and psychological health [4], [5], [6], [7]. Coping with these emotions at the current time is essential as it can affect their health well-being, the care they give to others while doing their job, and the well-being of the people they care about outside of work [8]. There are different coping strategies for instance, problem solving, information seeking, cognitive restructuring, emotional expression or ventilation, distraction, distancing, avoidance, wishful thinking, acceptance, seeking social support, and denial [9]. The most common are problem-focused coping and emotional focused coping. Problemfocused coping is the one that depends on changing or modifying the causes of stress and controlling them. This type can be effective when stressor is changeable or modifiable. In health-care system, this can be done through implementation of clear infection control guidance, effective education, and training of HCWs and availability of strict protective measures. While emotionally focused one can be effective when stressor is unchangeable and depends on wishful thinking, emotional release, family support, physical exercises, talking or meditation, reading, and hobbies [10], [11], 
[12], [13]. It was found that the more active problemfocused and control-oriented coping strategy, the less mental health symptoms and higher well-being. On the contrary, emotion-focused strategies as well as escapeoriented or avoidant strategies are generally associated with poor mental health and unwell-being [14].

The aim of the current work is to explore the different stress coping strategies that reduce stress among some critical care medicine physicians working in isolation hospitals in Egypt during the COVID-19 pandemic. This knowledge is crucial to help policymakers to plan good educational and support systems for them.

\section{Design and Methods}

\section{Study design and setting}

This is an exploratory study that used a qualitative approach. We conducted the study among critical care medicine physicians at two COVID-19 isolation hospitals across Cairo Governorate that was assigned by the government to deal only with COVID-19 cases. The study was performed in accordance with the CORE-Q reporting guidelines for qualitative studies [14].

\section{Sample size and sampling technique}

We chose participants using a purposive sampling technique. The study population were physicians who were working at COVID-19 isolation hospitals where they were continuously exposed to COVID-19 patients. The semi-structured interviews were conducted from March 20, 2020, to April 15, 2020 during the COVID-19 First wave. Initially, 41 participants consented to be part of the study, however, 9 of them refused for some personal reasons. The last sample comprised 32 participants. We continued the interviews until we reached data saturation, where no new themes, subthemes, or explanations emerged from the interviews [15]

\section{Data collection tools}

The researchers sent invitations to participants to explore the factors that helped them to reduce stress while dealing with the COVID-19 pandemic and to give their suggestions for strategies to cope with stress to deal with the COVID-19 pandemic and maintain resilience in stress working environments. A semi-structured interview guide was used that comprised open-ended questions, and minor revisions were completed after a pilot test. Systematic reviews of the literature were used to develop interview guides [4], [16] (Table 1).
Table 1: Structured interview guide

Background characteristics

What works best to prevent stress among health care workers while working during COVID-19 era?

Factors reducing stress in the work environment.

How can you cope with different stress during COVID-19 era?

To what extent do you feel current hospital training adequately addresses the learning needs of how to deal with COVID-19?

What were your thought or actions to reduce stress?

What are the policies and procedures within your health-care facility to deal with COVID-19-related stress?

Furthermore, the researcher asked probing questions for further clarification of the answers given by participants

The guide was pilot tested among a sample of three participants testing for the feasibility of the study, the clarity of the questions, the required duration to get the needed information, and the required modifications in the guide were done based on the pilot test results.

Interviews were performed and completed with physicians either face to face or through telephone for up to $30 \mathrm{~min}$ by one researcher who has ample experience in qualitative interview and had worked on many qualitative studies on resilience and protective factors of various communities. All interviews were audio recorded and transcribed.

\section{Data analysis}

We based data processing on the thematic content analysis technique, which aims to get descriptions of the message content using a systematic and objective procedure. This method has been used to generate a rich and comprehensive understanding of complex phenomena [16], [17]. The participants' quotations were used to clarify the meaning of the themes and summaries. The analysis was carried out independently by two authors M.R and S. E. They read the transcript multiple times, condensed, and made meaningful statements, and devised themes and subthemes. For demographic data, the researchers used Excel 2010. To summarize, the quantitative variables using mean and standard deviation, and the qualitative variables using number and percentages

\section{Ethical approval}

The Ethical Review Committee at Cairo University revised and approved the study protocol. All the study participants were treated according to the Helsinki Declaration of biomedical ethics. A written informed consent from each participant was obtained after proper orientation regarding the study objectives (whether electronically or handwritten). Data confidentiality and informants' identity were maintained throughout the study. For each participant, we will use: "l" (interviewee) followed by a number according to the chronologic order of the interviews (I01, I02, I03... .I13). 


\section{Results}

The participants who accepted the invitation to participate in the study were 32 participants. The mean age of them was $35 \pm 1.5$ years old and they had 3-12 years of work experience. About two-thirds of them were women $(66 \%)$. More than half of the study participants were married $(62.5 \%)$ and $60 \%$ of them had children. Most of the interviewed participants were living with their families at the time of the COVID-19 pandemic.

The transcripts of the interviews displayed factors reducing stress and stress coping strategies within the thematic analysis as stated by study participants.

\section{Factors reducing stress in the work environment}

\section{Training on providing care safely}

All interviewees mentioned that training HCWs on providing care safely helped to reduce their fear and stress and to become more confident about the care that they provide. Effective training must be associated with the supply of essential resources such as PPE "PPEs (especially disposable scrubs, gloves, face shields, and highly efficient masks) and hand sanitizer," one physician said. All interviewees universally agreed that providing PPE and clear infection control guidelines to physicians reduced stress. One physician mentioned that "Considering every patient admitted to the hospital as having COVID-19 infection and using full protective measures even if the patient was COVID-19 negative overcomes fear and stress."

\section{Familiarity with the available information}

The fear of the disease and uncertainty about how to protect themselves from infection as well as care for patients were increased by lack of knowledge and misconceptions about COVID-19 among health workers. This was slowly improved overtime, as physicians were learned more about COVID-19 and learned methods to protect themselves. Most of the interviewees stated, "When we started learning about COVID-19, it became better."

\section{Work climate}

All participants mentioned that working together on the first line, in addition to feeling a positive attitude from colleagues in their departments, had an important role in reducing stress during the pandemic. One of the interviewed personnel reported that "Sharing jokes among colleagues was one of the most stress coping strategies." Giving psychosocial support to HCWs in health-care delivery centers was beneficial for reducing stress. "Social workers and Psychiatrists helped us to deal with the stigma of being a HCW during a pandemic," one physician said.

\section{Personal stress coping strategies among frontline physicians and interns}

\section{Psychological adjustment}

Most of the interviewees stated that taking breaks from watching the news to engage in other relaxing and meaningful activities, such as reading or listening to news stories mainly on social media, helped them cope with the stress. Other strategies included trying to be busy at home doing activities to keep their minds away from COVID-19, getting enough rest, finding spare time during work or between shifts, and eating meals (ideally eating healthy food and eating on a schedule). One physician mentioned that "Making a regular habit of stepping away from my computer and smartphone from time to time is very important. When returning online, I tried to focus on information from reputable sources, not just sources in my social media feed."

"I listen to music, or practice yoga when I rest, and feel relaxed after crying," others stated. Another intern said: "I feel good using relaxation techniques such as prayers, sports, exercises, talking to, and motivating myself to face the COVID-19 pandemic with a positive attitude."

- $\quad$ Develop a support system (family and peer support)

Almost all the interviewees stated that they talked with family and friends to relieve stress and get support. Physicians highlighted many forms of support within their teams "Everyone is very welcoming and friendly. Professional colleagues will calm me and in turn I can calm new colleagues," "Everyone is willing to do more work to rest others. Colleagues are particularly united and help each other." The supportive environment was a stress relieving factor, especially at times of work overload.

All the interviewees mentioned that they felt that the collective power and the team bond was stronger. "If someone become uncomfortable, everyone will care of him and work for him," and "We encourage each other. It doesn't feel like I'm fighting alone, I'm not afraid." One physician said that "Feeling supported and being part of a united team can protect against the effects of stressful or traumatic experiences. And finally seeking professional help if symptoms persist or worsen over time is a must."

\section{Limit the consumption of news}

Consumption of news is limited by confining the information that is received to reputable sources 
and setting a time for when the news can be checked. Most of the interviewees reported that "Hearing about the pandemic repeatedly can be upsetting."

\section{Religion}

Many of the interviewees stated that their religious beliefs encouraged them to cope with the stress of seeing infected colleagues dying during the COVID-19 pandemic.

\section{Discussion}

In the current study, we explored the factors that helped Egyptian physicians to reduce stress while dealing with the COVID-19 pandemic as well as the different coping strategies they used. Our results showed that the training provided to the physicians on how to take all protective measures while caring for infected patients was an essential factor in reducing stress. Training HCWs to face a new situation have been reported in previous outbreaks and pandemics to decrease stress and improve the psychological wellbeing among the HCWs [18]. It sounds logical that we are more comfortable with things we already know and feel competent about, especially when the training defines the individual role of the managing teams and removes ambiguity. Efficient training would increase the cure rate and decrease deaths, which were also reported to reduce stress among participants.

Application of protective measures and strict implementation of infection control policy are very important during this pandemic because they provide the only way to prevent an infection that, as yet, has no cure. The availability of protective equipment and disinfectants in working places along with efficient training on their proper use was also highly rated stress-relieving factors. This matched the results from many previous and current reports [8], [12], [19]. Assuring safety is an important factor that promotes productivity, especially when this alleviates fear and anxiety of transmitting infections to other colleagues, family members, or friends.

Knowledge about the new pandemic is still in its infancy. This opened the door to misconceptions and even myths, which increase anxiety and stress among health professionals who are expected to deal with the medical consequences of the infection and with its cultural effects, requiring them to spread awareness and correct concepts among other colleagues as well as the public. Thus, it was important to attain all the available knowledge about the virus and, as reported by the participants, it was a good factor to relieve their stress. In this regard, participants appreciated the role of health authorities to provide up-to-date information and to keep them well informed.
It has been shown that the work climate has an enormous effect on the mental health of individuals, so maintaining higher spirits with positive attitudes among colleagues was a crucial factor in alleviating anxiety [4]. These strategies were reported to be useful among our study participants who even reported the effect of a sense of humor and telling jokes as a mood changer. They also mentioned that providing HCWs with psychological and social support were extremely needed and appreciated, and the support makes one more resilient. Similarly, a previous study done by Xiao et al. reported that social support for HCWs reduced their stress and anxiety, improved their quality of sleep, and helped them to maintain a higher degree of self-efficacy and stable emotions [20]. Moreover, the sympathy, understanding, and good relationships among colleagues strengthened motivation and enhanced organizational commitment [21].

Our participants stressed the need for psychological support services, which were relatively lacking at the time of the interviews, to be provided. Others highlighted the role of psychiatrists to overcome the stigma. The role of psychological support and interventions has been nicely illustrated in previous work by Brooks et al. in 2018 [22].

Limiting exposure to news, mass media, and social media was among the successful strategies for reducing stress among our participants. They indicated that it was a source of negative energy and irritability and obtaining information selectively from trusted sources was very important. This resonates with the WHO recommendations about minimizing exposure to COVID-19 news, and to be selective about what could be useful for protection and prevention from trusted sources [23].

Many participants emphasized the role of religion and beliefs to make them accept and be psychologically prepared for disastrous events if they occur, such as intubating a colleague or seeing a loved one dying from the infection. The effect of religion and spiritual intervention has been thoroughly discussed in the literature. Researchers reported its effect in alleviating anxiety and reducing perceived stress, and they admitted that it plays a role as a coping strategy among HCWs, especially nurses [24], [25]. Although this factor cannot be applied universally because of different religious and spiritual orientations worldwide, it is still a well-known factor in our Middle Eastern societies.

In addition, some participants stated that taking a rest between shifts, eating healthy food, exercising, or even crying can attenuate stress and relieve its effects. These were also included as stress reducing factors in the previous studies [12], [18].

A good group of family, friends, and colleagues plays a great role in reducing stress. Our interviewees stated that talking with their families and friends 
together with support from professional colleagues and teammates relieved stress and facilitated working under stressful conditions. This was confirmed in many studies from previous outbreaks and in the current pandemic [3], [8], [12], [22].

In summary, physicians and interns in Egypt have almost the same strategies to reduce stress as their counterparts worldwide, and they practice coping strategies that are either related to work environment or personal life. These strategies are mainly based on safety and support.

Safety is mainly related to organizations that are responsible for providing adequate training and health education on safe practices and better job security, occupational stress coping, and managing complex professional practice situations. Support is also provided by the organizations, through leaders and decision-makers, as well as by peers for better working conditions and allowing flexible scheduling, role clarity, and good communication skills.

However, social support is needed and provided mainly by families, friends, and the public.

Having short breaks, limiting exposure to the news, expressing emotions, adopting positive attitudes, seeking updated knowledge, positive thinking, increasing one's self-confidence, practicing relaxation, prayer, and spiritual interventions are personal strategies that need to be encouraged because they have been shown to be effective.

\section{Conclusion}

The present study revealed different stressreducing strategies that were recommended by the participants. These strategies are mainly based on organizational factors including providing in depth knowledge and PPE with sufficient training on infection control, individual factors mainly building emotional resilience hand in hand with public support. Implementing and promoting these strategies by organizations and individual HCWs are expected to reduce their stress and consequently promote productivity and endurance throughout the current pandemic.

\section{Limitations}

The limitations of this study are related to the fact that only some physicians at specialized hospitals in Egypt were interviewed, However, we can assure that these limitations do not affect the results because this is a qualitative approach to explore the factors that reduce stress as well as different strategies to cope with stress that is used by health-care professionals in Egypt during the COVID-19 pandemic, with no intention to generalize the results beyond the scope of the study sample. Further studies using different research methods including quantitative as well as qualitative that involve different institutions are needed to explore new stress coping strategies that can be of help during epidemics and pandemics.

\section{Authors' contributions}

E. S was the primary author of the article and contributed to the content regarding the methodology and data analysis, M.R contributed to data analysis and writing. L. E contributed to the content regarding data collection and the discussion. A.T contributed to the content regarding the introduction and discussion. All authors participated in developing the final version of the article.

\section{Acknowledgments}

We acknowledge all colleagues who participated in our study.

\section{References}

1. World Health Organization. WHO Director-General's Opening Remarks at the Media Briefing on COVID-19. Geneva: World Health Organization; 2020. Available from: https://www. who.int/dg/speeches/detail/who-director-general-s-openingremarks-at-the-media-briefing-on-covid-19-11-march-2020. [Last accessed on 2020 Sep 17]. https://doi.org/10.1093/ ww/9780199540884.013.u44226

2. Taiwan Centers for Disease Control: Coronavirus Disease 2019 (COVID-19); 2019. Available from: https://www. cdc.gov.tw/en/category/listcontent/bg0g_vu_ysrgkes_ krudgq?uaid=0nazwpxdbniapovjhwrgoq. [Last accessed on 2020 Apr 26]

3. World Health Organization. Global Alert and Response (GAR): Novel Coronavirus Infection in the United Kingdom. Geneva, Switzerland: World Health Organization; 2020. Available from: http://www.emro.who.int/ index.html. [Last accessed on 2020 Apr 26]. https://doi.org/10.26719/2015.21.6.379

4. Cai H, Tu B, Ma J, Chen L, Fu L, Jiang Y, et al. Psychological impact and coping strategies of frontline medical staff in Hunan between January and March 2020 during the outbreak of Coronavirus disease 2019 (COVID-19) in Hubei, China. Med Sci Monit. 2020;26:e924171. https://doi.org/10.12659/msm.924171 PMid:32291383

5. Tam CW, Pang EP, Lam LC, Chiu HF. Severe acute respiratory syndrome (SARS) in Hong Kong in 2003: Stress and psychological impact among frontline healthcare workers. Psychol Med. 2004;34(7):1197-204. https://doi.org/10.1017/ s0033291704002247

PMid:15697046

6. Lee SM, Kang, WS, Cho A, Kim T, Park JK. Psychological impact 
of the 2015 MERS outbreak on hospital workers and quarantined hemodialysis patients. Compr Psychiatry. 2018;87:123-7. https://doi.org/10.1016/j.comppsych.2018.10.003 PMid:30343247

7. Zhu Z, Xu S, Wang H, Liu Z, Wu J, Li G, et al. COVID-19 in Wuhan: Immediate Psychological Impact on 5062 Health Workers, MedRxiv; 2020.

8. Lai J, Ma S, Wang Y, Cai Z, Hu J, Wei N, et al. Factors associated with mental health outcomes among health care workers exposed to Coronavirus disease 2019. JAMA Netw Open. 2020;3(3):e203976. https://doi.org/10.1001/ jamanetworkopen.2020.3976 PMid:32202646

9. Dubow EF, Rubinlicht M. Encyclopedia of Adolescence. Amsterdam: Elsevier; 2011. p. 109-18.

10. Schaufeli WB. Job stress, coping with. In: International Encyclopedia of the Social and Behavioral Sciences. Amsterdam: Elsevier; 2001. p. 7984-7. https://doi.org/10.1016/ b0-08-043076-7/03844-4

11. Jordan TR, Khubchandani J, Wiblishauser M. The impact of perceived stress and coping adequacy on the health of nurses: A pilot investigation. Nurs Res Pract. 2016;2016:5843256. https://doi.org/10.1155/2016/5843256

\section{PMid:27882246}

12. Khalid I, Khalid TJ, Qabajah MR, Barnard AG. Healthcare workers emotions, perceived stressors and coping strategies during a MERS-CoV outbreak. Clin Med Res. 2016;14(1):7-14. https://doi.org/10.3121/cmr.2016.1303 PMid:26847480

13. Gulwadi GB. Seeking restorative experiences: Elementary school teachers' choices for places that enable coping with stress. Environ Behav. 2006;38:503-20. https://doi. org/10.1177/0013916505283420

14. Booth A, Hannes K, Harden A, Noyes J, Harris J. COREQ (consolidated criteria for reporting qualitative studies). In: Guidelines for Reporting Health Research: A User's Manual. Oxford, UK: John Wiley \& Sons Ltd.; 2014. p. 214-26. https:// doi.org/10.1002/9781118715598.ch21

15. Fusch PI, Ness LR. Are we there yet? Data saturation in qualitative research. Qual Rep. 2015;20(9):1408-16. Available from: http://www.nova.edu/ssss/qr/qr20/9/fusch1.pdf. [Last accessed on 2020 Apr 14].

16. Braun V, Clarke V. Using thematic analysis in psychology. Qual Res Psychol. 2006;3:77-101.

17. Sun $N$, Wei $L$, Shi $S$, Jiao $D$, Song $R$, Ma $L$, et al. A qualitative study on the psychological experience of caregivers of COVID-19 patients. Am J Infect Control. 2020;48(6):592-8 PMid:32334904

18. Maunder RG, Lancee WJ, Mae R, Vincent L, Peladeau N Beduz MA, et al. Computer-assisted resilience training to prepare healthcare workers for pandemic influenza: A randomized trial of the optimal dose of training. BMC Health Serv Res. 2010;10(1):72. https://doi.org/10.1186/1472-6963-10-72 PMid:20307302

19. Ly T, Selgelid MJ, Kerridge I. Pandemic and public health controls: Toward an equitable compensation system. J Law Med. 2007;15(2):296-302. PMid: 18035845

20. Xiao H, Zhang Y, Kong D, Li S, Yang N. The effects of social support on sleep quality of medical staff treating patients with Coronavirus disease 2019 (COVID-19) in January and February 2020 in China. Med Sci Monit. 2020;26:e923549. https://doi. org/10.12659/msm.923549

PMid:32132521

21. Baeriswyl S, Krause A, Elfering A, Berset M. How workload and coworker support relate to emotional exhaustion: The mediating role of sickness presenteeism. Int J Stress Manag. 2017;24:5273. https://doi.org/10.1037/str0000018

22. Brooks SK, Dunn R, Amlôt R, Rubin GJ, Greenberg N. A systematic, thematic review of social and occupational factors associated with psychological outcomes in healthcare employees during an infectious disease outbreak. J Occup Environ Med. 2018;60(3):248-57. https://doi.org/10.1097/ jom.0000000000001235 PMid:29252922

23. World Health Organization. Mental Health and Psychosocia Considerations during the COVID-19 Outbreak. Geneva, Switzerland: World Health Organization; 2020. Available from: https://www.who.int/docs/default-source/coronavi ruse/mentalhealth-considerations.pdf. [Last accessed on $2020 \mathrm{Apr}$ 01]. https://doi.org/10.1037/e400972004-001

24. Habibian N, Ahmadi R, Vashian A, Mortazavi SM, DadkhahTehrani T. Investigating the correlation between the life and religious attitudes with psychological well being in nurses working in health centers at Qom universities of medical sciences in 2014. Mediterr J Soc Sci. 2015;6(6):168-74. https:// doi.org/10.5901/mjss.2015.v6n6s4p168

25. Brass $\mathrm{E}$. How mindfulness can benefit nursing practice. Nurs Times. 2016;112(18):21-3.

PMid:27344898 